\title{
Palmo-Plantar Hyperhidrosis: Does Drainless One-port Thoracoscopic Sympathectomy (DOTS) help?
}

\author{
Mohammed El-Hag-Aly ${ }^{1}$, Rafik Soliman ${ }^{1}$, Nehad Zaid ${ }^{1}$, Mahmoud Eldesouky ${ }^{1}$, Mohamed \\ Bakoush $^{1}$, and Mohamed Hagag ${ }^{1}$ \\ ${ }^{1}$ Menoufia University Faculty of Medicine
}

June 2, 2020

\begin{abstract}
Objectives: Primary hyperhidrosis ( $\mathrm{PH}$ ) still impacts negatively the patients' quality of life (QoL). Progressively, it leads to poorer QoL regardless of gender; finally, it ends up causing psycho-social and professional embracement to patients. The thoracoscopic sympathectomy (TS) has been used safely and effectively for control of palmar hyperhidrosis; but it is still questionable in palmo-plantar type. We assessed the benefits of drainless one-port thoracoscopic sympathectomy (DOTS) in palmo-plantar and palmar hyperhidrosis. Methods: This prospective study comprised 213 consecutive patients with $\mathrm{PH}$ who underwent bilateral simultaneous DOTS. We used the Hyperhidrosis Disease Severity Scale (HDSS) scoring system for assessment of quality of life pre- and post-operatively. Follow up continued for one-year interval for quality of life, recurrence, and compensatory hyperhidrosis. Results: All patients experienced immediate complete resolution of hyperhidrosis postoperatively. Recurrence encountered in one patient during first 6 months. All patients had improved quality of life postoperatively; but at the end of 1-year follow up, $2.8 \%$ of patients were still suffering moderate to severe impaired QoL. Mean hospital stay was $31.79 \pm 17.5$ hours. We found significant longer hospital stay in palmar group than palmo-plantar group. There was no significant difference between both groups in neither operative time, recurrence, nor compensatory hyperhidrosis. Conclusions: DOTS offers better quality of life for patients with primary hyperhidrosis even the palmo-plantar type. The palmo-plantar type benefits similarly to the palmar type. In palmo-plantar hyperhidrosis, we should rethink again about post-operative plantar hyperhidrosis; it is accepted redistribution compensatory hyperhidrosis rather than a miserable recurrence.
\end{abstract}

\section{Introduction:}

Hyperhidrosis is a sweat glands' benign functional disorder. Usually, it presents by heat or emotional stimulated excessive sweating beyond the physiological needs. Moreover, it causes psycho-social and professional embracement that negatively impacts the quality of life (QoL). [1-3] Hyperhidrosis may be primary or secondary. The etiology of primary hyperhidrosis $(\mathrm{PH})$ is still unknown. It affects about 0.6 to $1 \%$ in the general population. The most usual sites or domains of hyperhidrosis are the hands in $25 \%$ of cases, armpit in $20 \%$ and both in $55 \%$, while plantar hyperhidrosis noted in $45 \%$ of cases. [2, 4]

Management of those patients includes many medications as topical medication and botulinum toxin injection. [5] In 1920s, the trans-thoracotomy surgical treatment started to appear in medical practice with significant patient morbidity so, it failed to generate widespread acceptance. [6] Evolving thoracoscopy approach enhanced the thoracoscopic sympathectomy to become the surgical technique of choice for treating $\mathrm{PH}$. This minimally invasive procedure is simple and safe, with a high success rate with low morbidity and short hospital stay.[7] Although being an effective method for management of $\mathrm{PH}$, it has some complications such as compensatory hyperhidrosis $(\mathrm{CH})$, pneumothorax, Horner's syndrome, post-operative pain and recurrence. $[8,9]$

\section{Materials and Methods:}


In our prospective study from April 2018 to August 2019, we enrolled 213 consecutive patients diagnosed as primary hyperhidrosis. We managed the patients in the Cardiothoracic Surgery and Vascular Surgery departments, Menoufia university hospitals. The Ethical committee of Menoufia Faculty of Medicine approved data collection and waived the need for patient consent.

We used The Hyperhidrosis Disease Severity Scale (HDSS) scoring system to evaluate patients' quality of life throughout the study. We included patients with moderate to severe quality of life impairment; those patients are patients who scored 3 or 4 in the scale. They were managed by bilateral simultaneous drainless one-port thoracoscopic sympathectomy (DOTS), starting by right side then left one in the same session.

Anesthesiologist used general anesthesia with double lumen endotracheal tube to allow the single lung ventilation. Positioning in $45^{\circ}$ anti-Trendelenburg supine position with both arms abducted at $90^{\circ}$ enhanced the both sides to be operated on same session without repositioning. It ended up saving more time.

We operate with only one skin incision. In males, the incision was in $3^{\text {rd }}$ or $4^{\text {th }}$ space anterior axillary line; while in females, it was placed under mammary crease for cosmetic purpose. Through this single opening, we introduce 10-mm camera and electrocautery endoscopic spatula; no insufflator was required.

Parietal pleura overlying the chain was incised using electrocautery; then the sympathetic chain was divided by 5 seconds continuous cautery at top and bottom of R2, R3, R4. The lateral aspect of each rib was cauterized for 1-2 cm to ensure a complete cut of any Kuntz's inter-neuronal connections; if left uncut, it may cause failure of the sympathetic denervation.

With coordination with anesthesiologist, lung expansion was observed under direct vision using continuous positive pressure for a few seconds at the end of the procedure. One or two stitches were applied to close the port site; the other side was operated afterwards using the same technique.

Post-operatively, follow-up of patients in the recovery room after being extubated was continued till full recovery from anesthesia. Normal equality of air entry and oxygen saturation were necessary for discharge to ward. Paracetamol or non-steroidal anti-inflammatory drugs (NSAIDS) were the drugs we used for pain control. We performed a chest x-ray film to detect post-operative hemothorax or pneumothorax. We recorded neurological problems (as: Horner's syndrome) and hospital stay (in hours).

Follow-up in the outpatient clinic was after one week, one month, 6 months, and 1 year after discharge. Every visit, we checked for: improvement of hyperhidrosis (using HDSS Score), recurrence, CH (site and severity), and wound infection.

Statistical analysis: Collected data was expressed as mean and standard deviation $(\mathrm{M} \pm \mathrm{SD})$ or number and percent (n, \%). Comparison between the two groups was performed using t test, Chi square analysis $\left(\chi^{2}\right)$, or Fischer's exact test when appropriate. The data were considered significant if P-value was less than 0.05. Statistical analysis was performed with the aid of the IBM Statistical Package for Social Science (SPSS) version 20 (IBM corporation, Chicago, IL, USA).

\section{Results:}

Between April 2108 and August 2019, 213 patients (61.5\% females) underwent DOTS. They were 20.83土4.12 years old. $37.1 \%$ had plantar domain of hyperhidrosis. Patient characteristics are enlisted in Table 1.

Average overall hospital stay was $31.79 \pm 17.5$ hours entailing the whole patient's stay within hospital from admission till discharge. Unfortunately, some patients suffered few complications (Table 2) as pneumothorax in $6(2.8 \%)$ patients from which $2(0.9 \%)$ patients only require intercostal chest tube (ICT) insertion. No Horner's syndrome cases, wound infection, nor mortality were encountered in our series.

Compensatory hyperhidrosis started to appear in our patients in 6-months and one-year follow-up visits; the overall incidence was $35.7 \%$ of patients. Domains and percentages are grouped in Table 2. Moreover, we had one patient presented with recurrence only in one side on the one-month follow-up visit $(0.5 \%)$, he refused 
to undergo a redo procedure again because it was affecting his left non-dominant limb so according to his words "it is not affecting my daily life to force me to go for surgery again".

All patients experienced immediate complete resolution of hyperhidrosis postoperatively; however, during the 1-year follow-up interval, $1(0.5 \%)$ patient was still suffering moderate impaired QoL at 6-months follow-up compared to $6(2.8 \%)$ patients at one-year follow-up (Table 2).

Comparing the 79 (37.1\%) patients with plantar domain (palmo-plantar group) to the remaining patients (palmar group) in the study group (Table 3), we detect significant higher body mass index (BMI) and longer hospital stay in palmar group. On the other side, we got no significant difference between both groups in operative time, recurrence, compensatory hyperhidrosis neither percent nor domains, nor postoperative HDSS score at 6-months and one-year intervals.

\section{Discussion:}

Currently, thoracoscopic sympathectomy (TS) is accepted as a standard treatment technique for PH. This minimally invasive approach has many benefits include reducing postoperative pain, shorter hospitalization, earlier recovery and return to work, and fewer complications. Both sides can be treated in the same sitting, thus avoiding readmission for a second procedure for the opposite side. [10]

The thoracoscopic approach allows clear delineation of the sympathetic chain and the ganglia including the collateral branches (Kuntz's nerves). Better visualization of the stellate ganglion and its preservation to avoid development of post-operative Horner's syndrome. [11]

As noted in many studies, the plantar domain affects considerable part of those patients of about 45\% [4]; while others reported incidence of plantar domain of 70 to $100 \%$ of patients with primary hyperhidrosis; latter researchers used extended technique of ablation from R3 to R12 to manage those cases [12]. In the Society of Thoracic Surgeons 2011 Expert Consensus for the Surgical Treatment of Hyperhidrosis [13], no recommendations for plantar domain management were noted. All of that arouse us to study such group of plantar hyperhidrosis patients with less invasive technique searching for acceptable outcome.

Our study included 213 patients diagnosed as primary hyperhidrosis and managed by DOTS approach. They had immediate improvement of hyperhidrosis without any mortality. There was no need for the placement of ports in any patient, and no conversion to open thoracotomy in our series.

Mean BMI in our study was $23.1 \pm 2.9$ going similarly with Wolosker n. et al. [14] study who reported mean BMI of $20.6 \pm 3$ and $21.9 \pm 2.55$ for their adolescent and adult groups. Repeatedly, different studies reported that results will not be satisfactory in patients with higher BMI, because of an increased prevalence of severe $\mathrm{CH}$ has been demonstrated in patients with a BMI higher than 25 [15]. Miller DL. et al. [16] in their study including 282 patients found that increased BMI was associated with increased in $\mathrm{CH}$. They explain this as heavier patients usually experience total body excessive sweating, rather than sweating in isolated areas. Patients with higher BMI have more difficulty with their thermoregulation. Again, Ishy A. et al. [17] in their Study including 40 patients found that individuals with a BMI more than 25 are predisposed to have a higher incidence of $\mathrm{CH}$.

Using the HDSS scoring for selecting patients, the patients scored 3 were $55.4 \%$ of study patients and those scored 4 were $44.6 \%$. Postoperatively, at one-year follow-up, dramatic improvement of patients' QoL was achieved; the severity score changed to $97.2 \%$ mild (score 1 in $93.9 \%$ and score 2 in $3.3 \%$ ) and $2.8 \%$ moderate to severe (score 3 in $1.9 \%$ and score 4 in $0.9 \%$ ). This was quite the same as Kuijpers M. et al. [18] in their study including 100 patients with bilateral thoracoscopic sympathectomy who reported mean HDSS score pre-operative of $3.69 \pm 0.47$ and mean post-operative HDSS of $1.06 \pm 0.34$ showing significant improvement. This throughout repeated studies proving that TS is an effective line in management of $\mathrm{PH}$ with improved QoL.

Despite that dramatic improvement in quality of life, we noticed the occurrence of compensatory hyperhidrosis. It manifests as excessive sweating in different areas of body appearing later after completely free interval 
of any excessive sweating. In our study, most of them were after 6 months; its overall incidence was $35.7 \%$ of patients. This is still the most common late complication, although its mechanism is still unclear. Our incidence of $\mathrm{CH}$ was lower than Prasad A. et al. [19] who had $63 \%$ post-operative $\mathrm{CH}$ after R3 resection; but it was higher than Ibrahim M. et al. [20] who reported $19 \%$ of their patients with CH. Their patients showed a gradually decreasing intensity over the follow-up period; they also resect from R2 to R4.

The DOTS procedure required operative time ranging 20-45 minutes with mean $35.03 \pm 4.1$ minutes. Nearly, Ibrahim M. et al. [20] noted in their study of 260 patients who had single stage bilateral thoracoscopic sympathectomy a mean operating time of $38 \pm 5.0$ minutes. On the other side, Kuijpers M. et al. [18] got a mean operative time of $74 \pm 12$ minutes for a bilateral sympathectomy; they used to perform their operation in lateral decubitus position, so more time was consumed in patient repositioning. When they shifted to beach chair position, they could perform the surgery on both sides without need for repositioning. This modification saved more time and showed significant reduction in their operation time to $47 \pm 18$ minutes (p $<0.001)$.

Post-DOTS pain was simple, it required only oral paracetamol or NSAIDs. No patient needed morphine. The DOTS approach causes less postoperative pain as post-thoracoscopic pain is usually related to the trauma of the thoracic wall caused by introducing the trocars into the intercostal space and periosteal injury.

On the interesting comparison of palmo-plantar patients to palmar ones, we found comparable groups with no significant difference regarding age, sex distribution, preoperative HDSS score, operative time, pneumothorax or recurrence.

Mean hospital stay was $35.23 \pm 21.14$ hours in palmar group. It was significantly longer than palmo-plantar group mean of $25.96 \pm 4.46$ hours $(\mathrm{p}=0.001)$; that may be due to higher rate of pneumothorax $(4.5 \%)$ and ICT insertion (1.5\%) in the palmar group.

Regarding $\mathrm{CH}$, it was not significant between the two groups with $34.4 \%$ in palmar group and $38 \%$ in palmoplantar group. Moreover, its distribution was not significant; the trunkal region was more affected followed by plantar region.

Precisely, the plantar CH was $12.7 \%$ in palmar group, which is nearly like $16.5 \%$ in palmo-plantar group. This finding enforced the possibility that patients with palmo-plantar hyperhidrosis would benefit from DOTS. Also, the postoperative plantar hyperhidrosis, reported in many previous studies as miserable recurrence with failure rates of 36-44\% [21], may be just redistributive acceptable $\mathrm{CH}$; if it occurs similarly in patients with different types of hyperhidrosis postoperatively.

At last, the overall outcome was satisfactory with patients HDSS score at one-year follow up of stage 1 in $94.8 \%$ of palmar group patients and $92.4 \%$ of palmo-plantar ones. Even with incidence of $\mathrm{CH}$, it still affects the quality of life less than $\mathrm{PH}$, as it is mild and much less affecting the professional or social life of the patients.

\section{Conclusion:}

DOTS offers better quality of life for patients with primary hyperhidrosis even the palmo-plantar type. The palmo-plantar type benefits similarly to the palmar type. In palmo-plantar hyperhidrosis, we should rethink again about post-operative plantar hyperhidrosis; it is accepted redistribution compensatory hyperhidrosis rather than a miserable recurrence.

\section{Author contributions:}

All authors were involved in conceptualization, investigation, methodology, resources, and visualization. Soliman R and Zaid $\mathrm{N}$ were involved in funding acquisition and project administration. El-Hag-Aly M, Soliman R, Zaid N, and Hagag M were involved in supervision and review and editing of the writing. El-Hag-Aly M, Eldesouky M, Bakoush M, and Hagag M were involved in data curation, formal analysis, software, Validation, and writing the original draft. 


\section{References:}

1. Wolosker N, Munia MA, Kauffman P, Campos JR, Yazbek G, Puech-Leão P. Is gender a predictive factor for satisfaction among patients undergoing sympathectomy to treat palmar hyperhidrosis? Clinics (Sao Paulo). 2010 Jun;65(6):583-6.

2. Gossot D, Kabiri H, Caliandro R, Debrosse D, Girard P, Grunenwald D. Early complications of thoracic endoscopic sympathectomy: a prospective study of 940 procedures. Ann Thorac Surg. 2001 Apr;71(4):1116-9.

3. Hajjar WM, Al-Nassar SA, Al-Sharif HM, Al-Olayet DM, Al-Otiebi WS, Al-Huqayl AA, et al. The quality of life and satisfaction rate of patients with upper limb hyperhidrosis before and after bilateral endoscopic thoracic sympathectomy. Saudi J Anaesth. 2019 Jan-Mar;13(1):16-22.

4. Karmota AG, Aboollo MF, Hafez BA, Shaker AA, Ghoneim B. Prospective randomized study comparing Video assisted thoracoscopic surgery (VATS) resection versus cautery for treatment of primary hyperhidrosis. Journal of the Egyptian Society of Cardio-Thoracic Surgery 2018; 26:318-323.

5. Dumont P, Denoyer A, Robin P. Long-term results of thoracoscopic sympathectomy for hyperhidrosis. Ann Thorac Surg. 2004 Nov;78(5):1801-7.

6. Dewey TM, Herbert MA, Hill SL, Prince SL, Mack MJ. One-year follow-up after thoracoscopic sympathectomy for hyperhidrosis: outcomes and consequences. Ann Thorac Surg. 2006 Apr;81(4):1227-32; discussion 1232-3.

7. Chung IH, Oh CS, Koh KS, Kim HJ, Paik HC, Lee DY. Anatomic variations of the T2 nerve root (including the nerve of Kuntz) and their implications for sympathectomy. J Thorac Cardiovasc Surg. 2002 Mar;123(3):498-501.

8. Youssef T, Soliman M. Unilateral sequential endoscopic thoracic sympathectomy for palmar hyperhidrosis: a proposed technique to overcome compensatory hyperhidrosis and improve plantar hyperhidrosis. J Laparoendosc Adv Surg Tech A. 2015 May;25(5):370-4.

9. Romero FR, Haddad GR, Miot HA, Cataneo DC. Palmar hyperhidrosis: clinical, pathophysiological, diagnostic and therapeutic aspects. An Bras Dermatol. 2016 Nov-Dec;91(6):716-725.

10. Du X, Zhu X, Wang T, et al. Compensatory hyperhidrosis after different surgeries at the same sympathetic levels: a meta-analysis. Ann Transl Med. 2018 Jun;6(11):203.

11. Vannucci F, Araújo JA. Thoracic sympathectomy for hyperhidrosis: from surgical indications to clinical results. J Thorac Dis. 2017 Apr;9(Suppl 3):S178-S192.

12. Elalfy K, Emile S, Elfeki H, Elmetwally A, Farag M, Gado W. Sequential Extended Thoracoscopic Sympathicotomy for Palmo-Axillo-Plantar Hyperhidrosis. Ann Thorac Surg. 2017 Oct;104(4):12001207.

13. Cerfolio RJ, De Campos JR, Bryant AS, et al. The Society of Thoracic Surgeons expert consensus for the surgical treatment of hyperhidrosis. Ann Thorac Surg. 2011 May;91(5):1642-8.

14. Wolosker N, Faustino CB, de Campos JRM, et al. Comparative analysis of the results of videothoracoscopic sympathectomy in the treatment of hyperhidrosis in adolescent patients. J Pediatr Surg. 2020 Jan 23. pii: S0022-3468(19)30837-1.

15. de Campos JR, Wolosker N, Takeda FR, et al. The body mass index and level of resection: predictive factors for compensatory sweating after sympathectomy. Clin Auton Res. 2005 Apr;15(2):116-20.

16. Miller DL, Bryant AS, Force SD, Miller JI Jr. Effect of sympathectomy level on the incidence of compensatory hyperhidrosis after sympathectomy for palmar hyperhidrosis. J Thorac Cardiovasc Surg. 2009 Sep;138(3):581-5.

17. Ishy A, de Campos JR, Wolosker N, et al. Objective evaluation of patients with palmar hyperhidrosis submitted to two levels of sympathectomy: T3 and T4. Interact Cardiovasc Thorac Surg. 2011 Apr;12(4):545-8.

18. Kuijpers M, Klinkenberg TJ, Bouma W, DeJongste MJ, Mariani MA. Single-port one-stage bilateral thoracoscopic sympathicotomy for severe hyperhidrosis: prospective analysis of a standardized approach. J Cardiothorac Surg. 2013 Nov 26;8:216.

19. Prasad A, Ali M, Kaul S. Endoscopic thoracic sympathectomy for primary palmar hyperidrosis. Surg Endosc. 2010 Aug;24(8):1952-7. 
20. Ibrahim M, Menna C, Andreetti C, et al. Bilateral single-port sympathectomy: long-term results and quality of life. Biomed Res Int. 2013;2013:348017.

21. Doolabh N, Horswell S, Williams M, et al. Thoracoscopic sympathectomy for hyperhidrosis: indications and results. Ann Thorac Surg 2004;77:410-4.

Table 1: Patient Preoperative Characteristics:

\begin{tabular}{lll}
\hline Variable & $\mathrm{n}=213$ & $\mathrm{n}=213$ \\
\hline Age, Mean \pm SD & $20.83 \pm 4.12$ & $20.83 \pm 4.12$ \\
Sex, n $(\%)$ & Sex, n (\%) & \\
Male & $82(38.5 \%)$ & $82(38.5 \%)$ \\
Female & $131(61.5 \%)$ & $131(61.5 \%)$ \\
BMI, Mean \pm SD & $23.1 \pm 2.9$ & $23.1 \pm 2.9$ \\
Area/Domain affected, n (\%) & Area/Domain affected, n (\%) & Area/Domain affected, n (\%) \\
Palmar & $213(100 \%)$ & $213(100 \%)$ \\
Axillary & $101(47.4 \%)$ & $101(47.4 \%)$ \\
Plantar & $79(37.1 \%)$ & $79(37.1 \%)$ \\
Pre-operative HDSS, n $(\%)$ & Pre-operative HDSS, n $(\%)$ & Pre-operative HDSS, n $(\%)$ \\
3 & $118(55.4 \%)$ & $118(55.4 \%)$ \\
4 & $95(44.6 \%)$ & $95(44.6 \%)$ \\
\hline
\end{tabular}

BMI: Body-Mass Index, HDSS: Hyperhidrosis Disease Severity Score

Table 2: Procedure Data:

\begin{tabular}{ll}
\hline Variable & $\mathrm{n}=213$ \\
\hline Operative time (min.), Mean \pm SD & $35.03 \pm 4.1$ \\
Hospital stay (hr.), Mean \pm SD & $31.79 \pm 17.5$ \\
Post-operative pneumothorax, n (\%) & $6(2.8 \%)$ \\
Post-operative ICT, n (\%) & $2(0.9 \%)$ \\
Compensatory Hyperhidrosis (CH), n (\%) & $76(35.7 \%)$ \\
Areas/Domains affected by CH, n (\%) & \\
Palmar & $1(0.5 \%)$ \\
Axillary & $1(0.5 \%)$ \\
Plantar & $30(14.1 \%)$ \\
Facial & $1(0.5 \%)$ \\
Trunkal & $75(35.2 \%)$ \\
Recurrence & $1(0.5 \%)$ \\
Postoperative HDSS Score at 6 months & \\
1 & $212(99.5 \%)$ \\
3 & $1(0.5 \%)$ \\
Postoperative HDSS Score at one year & \\
1 & $200(93.9 \%)$ \\
2 & $7(3.3 \%)$ \\
3 & $4(1.9 \%)$ \\
4 & $2(0.9 \%)$ \\
\hline
\end{tabular}

HDSS: Hyperhidrosis Disease Severity Score

Table 3: Palmar versus palmo-plantar outcome: 


\begin{tabular}{|c|c|c|c|}
\hline Variable & Palmar $(\mathrm{n}=134)$ & Palmo-Plantar $(\mathrm{n}=79)$ & P-Value \\
\hline Age, Mean \pm SD & $20.96 \pm 3.89$ & $20.61 \pm 4.48$ & 0.55 \\
\hline \multicolumn{4}{|l|}{ Sex, n $(\%)$} \\
\hline Male & $51(38.1 \%)$ & $31(39.2 \%)$ & 0.89 \\
\hline Female & $83(61.9 \%)$ & $48(60.8 \%)$ & \\
\hline BMI, Mean \pm SD & $23.64 \pm 3.25$ & $22.22 \pm 2$ & 0.001 \\
\hline Preoperative HDSS & Preoperative HDSS & Preoperative HDSS & Preoperative HDSS \\
\hline Score, n (\%) & Score, n (\%) & Score, n (\%) & Score, n (\%) \\
\hline 3 & $74(55.2 \%)$ & $44(55.7 \%)$ & 0.95 \\
\hline 4 & $60(44.8 \%)$ & $35(44.3 \%)$ & \\
\hline $\begin{array}{l}\text { Operation time, } \\
\text { Mean } \pm \text { SD }\end{array}$ & $35.43 \pm 3.99$ & $34.34 \pm 4.27$ & 0.06 \\
\hline $\begin{array}{l}\text { Hospital Stay, } \\
\text { Mean } \pm \text { SD }\end{array}$ & $35.23 \pm 21.14$ & $25.96 \pm 4.46$ & 0.001 \\
\hline Pneumothorax, n (\%) & $6(4.5 \%)$ & 0 & 0.08 \\
\hline $\mathrm{ICT}, \mathrm{n}(\%)$ & $2(1.5 \%)$ & 0 & 0.53 \\
\hline Recurrence, n (\%) & $1(0.7 \%)$ & 0 & 1 \\
\hline Compensatory & $46(34.3 \%)$ & $30(38 \%)$ & 0.59 \\
\hline \multicolumn{4}{|l|}{$\begin{array}{l}\text { Areas affected by } \mathrm{CH} \text {, } \\
\mathrm{n}(\%)\end{array}$} \\
\hline Palmar & $1(0.7 \%)$ & 0 & 1 \\
\hline Axillary & $1(0.7 \%)$ & 0 & 1 \\
\hline Plantar & $17(12.7 \%)$ & $13(16.5 \%)$ & 0.45 \\
\hline Facial & 0 & $1(1.3 \%)$ & 0.37 \\
\hline Trunkal & $45(33.6 \%)$ & $30(38 \%)$ & 0.52 \\
\hline Post-operative HDSS & Post-operative HDSS & Post-operative HDSS & Post-operative HDSS \\
\hline $\begin{array}{l}\text { Score at } 6 \text { months, } \mathrm{n} \\
(\%)\end{array}$ & $\begin{array}{l}\text { Score at } 6 \text { months, } \mathrm{n} \\
(\%)\end{array}$ & $\begin{array}{l}\text { Score at } 6 \text { months, } \mathrm{n} \\
(\%)\end{array}$ & $\begin{array}{l}\text { Score at } 6 \text { months, } \mathrm{n} \\
(\%)\end{array}$ \\
\hline 1 & $133(99.3 \%)$ & $79(100 \%)$ & 1 \\
\hline 3 & $1(0.7 \%)$ & 0 & \\
\hline Post-operative HDSS & Post-operative HDSS & Post-operative HDSS & Post-operative HDSS \\
\hline Score at 1 year, n (\%) & Score at 1 year, n (\%) & Score at 1 year, n (\%) & Score at 1 year, $\mathrm{n}(\%)$ \\
\hline 1 & $127(94.8 \%)$ & $73(92.4 \%)$ & 0.9 \\
\hline 2 & $4(3 \%)$ & $3(3.8 \%)$ & \\
\hline 3 & $2(1.5 \%)$ & $2(2.5 \%)$ & \\
\hline 4 & $1(0.7 \%)$ & $1(1.3 \%)$ & \\
\hline
\end{tabular}

BMI: Body-Mass Index, ICT: Intercostal Chest Tube, HDSS: Hyperhidrosis Disease Severity Score 